\title{
Nonspecific association of 2',3'-cyclic nucleotide 3 '-phosphodiesterase with the rat forebrain postsynaptic density fraction
}

\author{
Sun-Jung Cho ${ }^{1}$, Jae Seob Jung ${ }^{2}$ \\ Seung Chul Shin ${ }^{2}$, IngNyol Jin ${ }^{1}$ \\ Bok Hyun $\mathrm{Ko}^{2}$, Yunhee Kim Kwon ${ }^{3}$ \\ Haeyoung Suh-Kim ${ }^{4}$ and II Soo Moon ${ }^{2,5}$ \\ ${ }^{1}$ Department of Microbiology \\ College of Natural Sciences \\ Kyungpook National University \\ Daegu 702-701, Korea \\ ${ }^{2}$ Department of Anatomy and \\ Medical Institute of Dongguk University \\ College of Medicine, Dongguk University \\ Gyeongju 780-714, Korea \\ ${ }^{3}$ Department of Biology \\ Kyung Hee University \\ Seoul 130-701, Korea \\ ${ }^{4}$ Department of Anatomy \\ Brain Disease Research Center \\ School of Medicine, Ajou University \\ Suwon 442-721, Korea \\ ${ }^{5}$ Corresponding author: Tel, 82-54-770-2447; \\ Fax, 82-54-770-2414; E-mail, Fax:moonis@dongguk.ac.kr
}

Accepted 7 October 2003

Abbreviations: $\mathrm{BH}$, brain homogenate; $\mathrm{CNP}, 2^{\prime}, 3^{\prime}$-cyclic nucleotide 3'-phosphodiesterase; FB, forebrain; OG, n-octyl glucoside; OL, oligodendrocyte; PC, Purkinje cell; PSD, postsynaptic density; pTyr, phosphotyrosine; RT, room temperature; Sarc, N-lauroyl sarcosinate; Syn, synaptosome.

\footnotetext{
Abstract

The 2',3'-cyclic nucleotide 3 '-phosphodiesterase (CNP), a protein of unknown function in vivo, is abundantly expressed in myelinating glia in two is oforms, CNP1 and CNP2. In this study, immunoblot analysis showed that CNP1 is the major isoform in adult forebrain, and that both is oforms are included in the postsynaptic density (PSD) fraction and tyrosine-phosphorylated at the basal level. However, subcellular distribution and detergent extraction data showed that CNP is nonspecifically associated with the PSD fraction. Immunocytochemistry revealed that CNP is detected, in a
}

weak but punctate pattern, in dissociated rat hippocampal neurons of 3 days to 2 weeks in vitro. The CNP-positive punctae were distributed throughout soma and dendrites, and distinct from PSD95-positive ones. Immunoblot analysis indicated that CNP is also expressed in neuronal stem cell lines, HiB5 and F11. Interestingly, in addition to the known two isoforms, a new CNP isoform of MW $45 \mathrm{kDa}$ was expressed in these cell lines and was the major type of isoform in F11 cells. Taken together, our data suggest that CNP is expressed in the early stage of in vitro development and nonspecifically included in the adult rat PSD fraction.

Keywords: CNP; hippocampal cell; postsynaptic density fraction

\section{Introduction}

The 2',3'-cyclic nucleotide 3'-phosphodiesterase (CNP; EC 3.1.4.37) is expressed as two isoforms with an apparent molecular mass of $46 \mathrm{kDa}$ (CNP1) and 48 $\mathrm{kDa}$ (CNP2), which are produced by alternative ribosomal initiation at two different AUG codons, thus differing each other only by the 20-amino acid extension at the $\mathrm{N}$ terminus (O'Neill et al., 1997). Despite its lengthy history, relatively little is known about the function of CNP. In vitro, CNP specifically catalyzes the irreversible hydrolysis of $2^{\prime}, 3^{\prime}$-cyclic nucleotides to produce exclusively 2'-nucleotides (Tsukada and Kurihara, 1992). However, this enzymatic activity may not be relevant to its function in vivo, because physiologically relevant in vivo substrates with 2',3'-cyclic termini have not yet been elucidated. Indeed, recent findings by Lappe-Siefke et al. (2003) revealed that Cnp1, a gene that encodes both CNP1 and CNP2 in oligodendrocytes, is essential for axonal survival but not for myelin assembly. However, how CNP supports axons is not known.

The CNP is highly expressed in myelin-forming glial cells and, so far, widely used as a marker protein for oligodendrocytes (OL's) in the central nervous system (CNS) (for reviews see Vogel and Thompson, 1988). In addition to myelinating cells such as oligodendrocytes and Schwann cells, CNP is also expressed in various cell types such as chromaffin cell cul- 
tures (McFerran and Burgoyne, 1997), lymphocytes and retinal cells (Uyemura et al., 1972; Giulian and Moore, 1980; Weissbarth et al., 1981; Vogel and Thompson, 1988), liver cells (Dreiling et al., 1981), FRTL5 thyroid cells (Laezza et al., 1997; Bifulco et al., 2002), and muscle cells (Weissbarth et al., 1981), indicating more general cellular functions. Recently, we found that CNP is expressed in Purkinje cells (PC's) and unidentified PSD95-positive cells but not in granule cells (GC's) in cerebellar cell cultures, indicating that both CNP is expressed in some subpopulations of cerebellar cells in addition to OL (Cho et al., 2003). Expression of CNP in these cell types was patchy and was co-isolated with the postsynaptic density (PSD), a cytoskeletal specialization that has various functions for synaptic regulation (Siekevitz, 1985; Kennedy, 1997, 1998, 2000). Since immunoblot analysis indicated that CNP is also included in the forebrain PSD fraction, we characterized in detail its expression in hippocampal neurons and association with the forebrain PSD. Here, we show evidence that CNP is transiently expressed at a low level in hippocampal neurons in culture but does not colocalize with the PSD95, a marker for the PSD.

\section{Materials and Methods}

\section{Antibodies}

The mouse monoclonal antibodies; $\alpha-C N P$ (clone 11 $5 B$; Chemicon, Temecula, CA), $\alpha$-PSD95 family (clone K28/86.2; Upstate Biotechnology Inc., Lake Placid, $N Y$ ), and $\alpha$-pan-actin (clone C4, Bohringer Mannheim) were obtained as indicated. The rabbit polyclonal $\alpha$ CNP was generated using GST-CNP fusion protein (N-terminal 220 amino acids) and affinity-purified (Cho et al., 2003). The Alexa Fluor 488-conjugated goat anti-mouse IgG and Alexa Fluor 568-conjugated goat anti-rabbit $\lg G$ were purchased from Molecular Probes (Leiden, The Netherlands).

\section{Subcellular fractionation}

PSD fractions were prepared from adult rat (SpragueDawley, 200-250 gr) forebrain as previously described (Carlin et al., 1980; Cho et al., 1992). Briefly, synaptosomes were isolated from homogenates by sucrose step-gradient centrifugation and then extracted with $0.5 \%$ Triton $\mathrm{X}-100$ for $15 \mathrm{~min}$ at $4^{\circ} \mathrm{C}$. After centrifugation $(36,800 \mathrm{~g})$ for $45 \mathrm{~min}$ at $4^{\circ} \mathrm{C}$, pellets were resuspended in $40 \mathrm{mM}$ Tris- $\mathrm{HCl}(\mathrm{pH} \mathrm{8.0)}$ ). For detergent extraction experiments, 'One-Triton' PSD fractions were treated with $1.0 \%$ n-octyl glucoside $(O G)$, or $1.0 \%$ Triton $\mathrm{X}-100$, or with $3 \% \mathrm{~N}$-lauroyl sarcosine (Sarc) for $15 \mathrm{~min}$ at $4^{\circ} \mathrm{C}$ and then the pellet and supernatant fractions were separated by centrifugation at 201,800 $g$ for $1 \mathrm{~h}$ at $4^{\circ} \mathrm{C}$. Protein concentrations were determined using Advanced Protein Assay Reagents (Cytoskelecton, Denver) and double-checked by densitometric measurement of Coomassie-stained SDS-gel.

\section{Immunoblotting}

After SDS-PAGE, proteins were transferred to a nitrocellulose membrane and blocked overnight at $4^{\circ} \mathrm{C}$ in TTBS. Blots were incubated with primary antibodies [mouse monoclonal $\alpha$-CNP $(1: 2,000)$, rabbit polyclonal $\alpha$-CNP $(1: 5,000)$ or $\alpha$-PSD95 $(1: 2,000)]$ for $2 h$ at RT. Blots were rinsed with TTBS $(4 \times 20 \mathrm{~min})$ and the antigen-antibody complex was visualized with alkaline phosphatase-conjugated secondary antibodies according to the manufacturer's instructions (Roche, Germany). For quantification, blots were scanned, and the signal densities were measured with the $\mathrm{NIH}$ Scion Image Beta 4.0.2 software.

\section{Immunoprecipitation}

OG-P fractions (600 $\mu \mathrm{g}$ each) were brought to $2.5 \%$ SDS and boiled for $3 \mathrm{~min}$. The volume was adjusted to $1.0 \mathrm{ml}$ with RIPA buffer [10 $\mathrm{mM}$ Tris- $\mathrm{HCl}(\mathrm{pH} 7.4)$, $1.0 \mathrm{mM}$ EDTA, $150 \mathrm{mM} \mathrm{NaCl}, 1.0 \%$ Triton $\mathrm{X}-100$, $1.0 \%$ sodium deoxycholate, and $0.1 \%$ SDS (added after boiling step)]. Then, the affinity-purified rabbit anti-CNP antibody (1:50) was added. After incubation of the mixture for $2 \mathrm{~h}$ at $4^{\circ} \mathrm{C}$, protein A-agarose slurry (1:1) was added and further incubated for $2 \mathrm{~h}$. The agarose beads were washed with RIPA buffer $(5 \times 5$ $\mathrm{min}, 1.0 \mathrm{ml}$ each). After a brief spin, supernatant was discarded and SDS-gel sample buffer was added to the mixture. After the mixture was boiled for $3 \mathrm{~min}$ and centrifuged briefly, the supernatant was applied to SDS-gels.

\section{Dissociated culture}

Hippocampal cells were dissociated from embryonic day 18 (E18) rat (Sprague-Dawley) fetal brains according to Brewer et al. (1993). A pregnant rat was anesthetized on dry ice, and hippocampi were dissected, cut into small pieces $(2-3 \mathrm{~mm})$ and treated with $0.25 \%$ trypsin at $37^{\circ} \mathrm{C}$ for $2 \mathrm{~min}$. After washing 4-5 times (5 $\mathrm{ml}$ each) with Hank's balanced salt solution (HBSS) containing sodium pyruvate $(1 \mathrm{mM})$ and HEPES $(10 \mathrm{mM}, \mathrm{pH} 7.4)$, tissues were transferred to $1.0 \mathrm{ml}$ Hank's solution and cells were dissociated by trituration (5-6 times) using flamed Pasteur pipettes. Dissociated cells were collected by centrifugation and seeded at the density of 1,000-1,500 cells/ $\mathrm{mm}^{2}$ on poly-D-lysine coated coverslips which were preincubated in 24-well culture plates containing 300 $\mu \mathrm{l}$ of plating media [Neurobasal medium with B27 supplement, $0.25 \mathrm{mM}$ glutamax I, $25 \mathrm{mM}$ glutamate, 
and $25 \mathrm{mM}$ 2-mercaptoethanol (GibcoBRL)]. Cells were maintained in a humidified incubator $\left(5 \% \mathrm{CO}_{2}\right.$, $37^{\circ} \mathrm{C}$ ). One third of culture media was exchanged with feeding media [Neurobasal medium supplemented with B27, $0.25 \mathrm{mM}$ glutamax I] for $2 \sim 3$ days.

\section{Immunocytochemical labeling of dissociated cerebellar cells}

Cultures of dissociated hippocampal cells were grown on poly-D-lysine-coated coverslips as described above. The culture plate was placed on ice and the medium was replaced with ice-cold D-PBS. Coverslips were rinsed briefly with $4^{\circ} \mathrm{C}$ methanol and cells were fixed with methanol for $20 \mathrm{~min}$ at $-20^{\circ} \mathrm{C}$. Coverslips were washed with ice-cold D-PBS $(1 \times 15 \mathrm{~min})$ and incubated in the preblock solution $[0.05 \%$ Triton $X$ $100,5 \%$ normal goat serum in h-PBS $(20 \mathrm{mM}$ sodium phosphate buffer, pH 7.4, $450 \mathrm{mM} \mathrm{NaCl})]$ for $4 \mathrm{~h}$ at $4^{\circ} \mathrm{C}$. Primary antibodies [affinity-purified rabbit polyclonal $\alpha$-CNP (1:500), mouse monoclonal $\alpha$ PSD95 (1:500), or monoclonal a-pan actin $(1: 200)]$ were added and incubated overnight at $4^{\circ} \mathrm{C}$. After washes $(2 \times 10 \mathrm{~min})$ in the preblock solution on ice, coverslips were incubated with Alexa488-conjugated goat antimouse or Alexa568-conjugated goat anti-rabbit IgG (1:500; Molecular Probes Inc.) for $1 \mathrm{~h}$. After washes $(3 \times 20 \mathrm{~min})$ in the preblock solution, followed by in h-PBS $(3 \times 10 \mathrm{~min})$. Coverslips were mounted with DABCO mounting solution (100 mg of 1,4-diazabicyclo[2,2,2]octane in $1 \mathrm{ml}$ of $90 \%$ glycerol and $10 \%$ PBS, $\mathrm{pH} 7.4$ ), and viewed in a fluorescence microscope equipped with filter systems $\mathrm{B}$ and N2.1 (Leica Research Microscope DM IRE, Germany). Images were captured through a CCD camera (Photometrics Inc. Germany) connected to a computer using the QFluoro version V1.0b software (Leica Microsystems AG, Wetzlar, Germany) under a condition that there was no bleed-over from the Alexa 488 fluorescence into the 568 channel and vice versa, and processed with an Adobe Photoshop 5.0 software.

\section{Results}

\section{Co-isolation of CNP in the FB-PSD fraction}

Immunoblot analysis was carried out to understand the subcellular distribution of CNP in the forebrain. Homogenate $(\mathrm{BH})$, synaptosome (Syn) and PSD fractions were prepared from adult rat forebrain and subjected to immunoblot analysis with $\alpha$-CNP antibody. As shown in Figure 1A, both CNP1 and CNP2 isoforms were present in the forebrain homogenate $(\mathrm{BH})$. Densitometric measurement of the immunoblot signals indicated that the expression of CNP2 was only half of the CNP1 at most. This result indicates

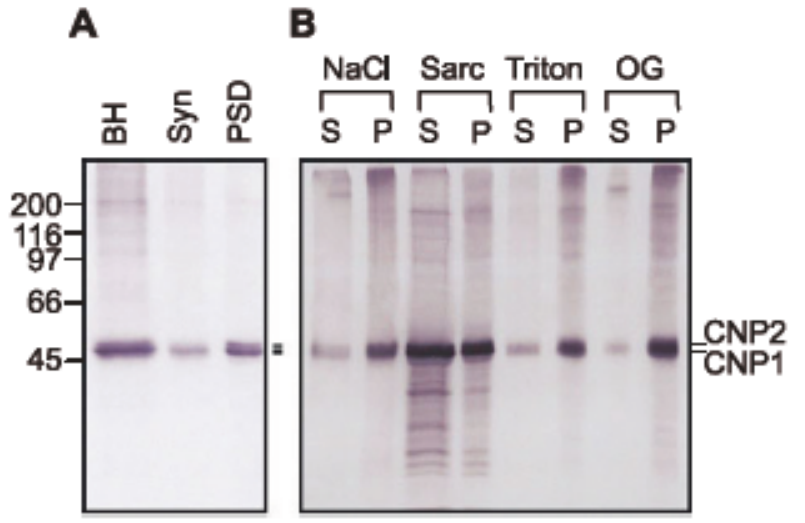

Figure 1. Immunoblot analysis for the expression of CNP in the rat forebrain. A, Subcellular distribution. Forty micrograms of homogenate (BH), synaptosome (Syn) and One-Triton PSD fraction from adult rat forebrain were electrophoresed on an $8 \%$ SDS-gel and immunoblotted with affinity-purified rabbit polyclonal $\alpha$-CNP antibody $(1: 5,000)$. Nole hat CNP1 (lower band) is much stronger than CNP2 (upper band) in all fractions. B, Extraction experiments. Forty micrograms of the OneTriton PSD fractions were extracled with $n$-octyl glucoside $(O G, 1 \%)$, or Trition X-100 (Triton, 1\%), or N-lauroyl sarcosinate (Sarc, 3\%), or $\mathrm{NaCl}(1.0 \mathrm{M})$. Soluble (S) and insoluble (P) fracions were electrophoresed on $8 \%$ SDS-gels and immunoblotted with $\alpha$-CNP monoclonal antbody. Posifons of CNP1 and CNP2 are marked by a hick and hin bar, respectively. Molecular sizes are shown in kilodaltons (KDa) on the left.

that CNP1 is the major form in the forebrain. When equal amounts of protein; $\mathrm{BH}, \mathrm{Syn}$, and PSD fractions were compared, CNP1 and CNP2 were not enriched in the PSD fraction and present $\sim 60 \%$ of the CNP in $\mathrm{BH}$, suggesting that the association of CNP with the PSD is nonspecific. To characterize the association properties of CNP with the PSD, the each component was solubilized with detergent. Weak detergents such as Triton $(1.0 \%)$ or OG $(1.0 \%)$ was not sufficiently able to solubilize the CNP leaving about $70 \sim 80 \%$ of CNP1 and CNP2 in the pellet (P) fractions, and $\mathrm{N}$-lauroyl sarconiate (Sarc, $3 \%$ ), a strong detergent, solublized greater amounts $(\sim 60 \%)$ of both CNP1 and CNP2 (Figure 1B). Also, the salt (1.0 M $\mathrm{NaCl}$ ), which will disrupt ionic interactions between proteins, was not efficient $(\sim 25 \%)$ in dissociating CNP from the PSD core. Taken together, these results suggest that the association of CNP with the FB-PSD fraction is probably nonspecific but fairly strong.

\section{Basal tyrosine-phosphorylation of CNP}

Next, a question of whether CNP represents the major phosphotyrosine-containing peptide in the band was raised because of earlier identification of the CNP in a phosphotyrosine-positive protein band in SDS-PAGE of cerebellar PSD fraction (Cho et al., 2003). A Coomassie-stained SDS-gel of $\alpha$-CNP immu- 


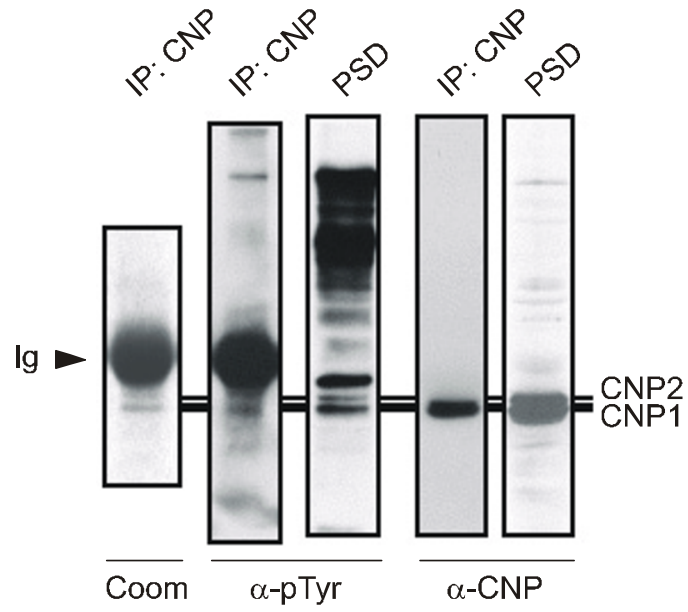

Figure 2. Basal tyrosine-phosphorylation of the CNP in the PSD fraction. CNP was immunoprecipitated from a PSD fraction (PSD) with affinity-purified rabbit polyclonal $\alpha-C N P$ antibody (IP: $\alpha$-CNP), electrophoresed on a 10\% SDS-gel and stained with Coomassie R-250 (Coom) or immunoblotted with either monoclonal $\alpha$-phosphotyrosine antibody 4 g10 ( $\alpha$-pTyr; 1:2,000, Upstate Biotechnology Inc.) or with $\alpha-C N P$. Forebrain PSD fraction (PSD) was included in the blot and used as references for immunoblot signals. Positions of CNP1 and CNP2 are marked by thick and thin bars, respectively, and the position of immunoglobulin $(\mathrm{lg})$ is marked by an arrowhead.

noprecipitate (IP) showed that polyclonal $\alpha$-CNP antibody precipitated 46 and $48 \mathrm{kDa}$ proteins (Figure 2, Coom., IP: CNP), which are recognized by a monoclonal $\alpha$-CNP antibody (Figure 2, $\alpha-C N P, I P$ : CNP) and corresponded well to the CNP's in the PSD lane (Figure 2, $\alpha$-CNP, PSD), indicating that CNP's were specifically immunoprecipiated. When the CNP1 and CNP2 IP's were immunoblotted with a monoclonal $\alpha$-phosphotyrosine antibody, they exhibited positive signals (Figure 2, $\alpha-p$ Tyr, IP: CNP), whose positions corresponded well to those in the PSD (Figure 2, $\alpha$-Tyr, PSD). However, the signal strength was much weaker than that in the PSD fraction, even though there are more CNP proteins in the IP:CNP lane than in the PSD one. Taken together, these results indicate that the CNP1 and CNP2 in the PSD fraction are phosphorylated on tyrosine at a basal level. Therefore, it should be noted that the major tyrosine-containing protein in the one-dimensional SDS-gel band may not be CNP.

\section{Transient expression of CNP in cultured hippocampal neurons}

Double-labeling of cultured hippocampal cells was done to further investigate possible expression of CNP in the PSD. Dissociated E18 hippocampal cells were grown on poly-D-lysine-coated coverslips in Neurobasal media supplemented with B27. In our
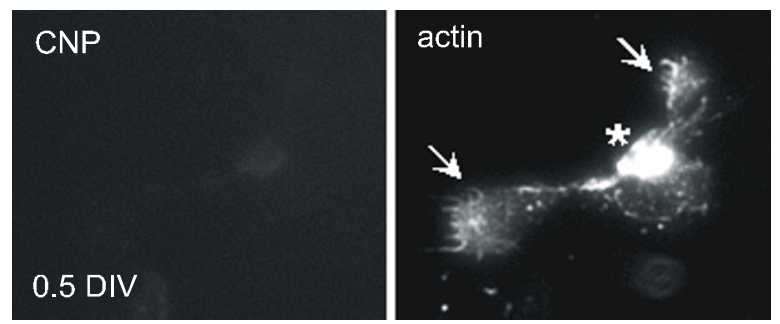

Figure 3. Immunocytochemistry showing no expression of CNP in 0.5 DIV hippocampal neurons. Hippocampal cells, dissociated on E18, were grown on coverslips in Neurobasal plus B27 supplement. At 12 $\mathrm{h}$ after plating, cells were fixed with methanol and double-stained with affinity-purified rabbit polyclonal $\alpha$-CNP $(1: 200)$ and mouse monoclonal $\alpha$-pan-actin (1:500). Primary antibodies were visualized by incubating with Alexa488-conjugated goat anti-mouse or Alexa568-conjugated goat anti-rabbit $\operatorname{lgG}$. Growth cones (arrows) and soma (asterisk) are marked in $\alpha$-pan-actin image (actin).

hands, PSD95, a postsynaptic marker, began to appear in cultured hippocampal neurons from 3 days in vitro (DIV). To see if CNP is expressed in the very early stage of in vitro morphological development, neurons were double-stained with $\alpha$-CNP and $\alpha$-actin antibodies at 0.5 DIV. Actively growing growth cones were evident in $\alpha$-actin staining (Figure 3, actin; arrows). The $\alpha-C N P$ staining showed that CNP was not expressed at this stage of development (Figure 3, CNP).

Neurons older than 3 DIV were double-stained with $\alpha$-CNP and $\alpha$-PSD95 antibodies. Since the latter is a marker protein for PSD (Cho et al., 1992), colocalization of the two proteins would implicate association of the CNP with the PSD and infer a function of CNP in spine formation. As shown in Figure 4, the levels of CNP expression in neurons were much lower than that of OL's but evident on $3,5,8,10$, and 12 DIV neurons. After about 2 weeks in vitro, its expression become very weak and its immunostain intensity was almost indistinguishable from control stains where primary antibodies were omitted. One interesting point to note is that $\alpha$-CNP staining was not homogenous but always punctate in both soma and dendrites (Figure 5, CNP). In order to find if $\alpha$-CNP punctae are same as those of $\alpha$-PSD 95, the two images were merged. One should note that the CNP signals were significantly enhanced and contrasted artificially in the photoShop program in this process. Merging the two images revealed that the $\alpha$-CNP punctae are not superimposed with those of $\alpha$-PSD95 (Figure 5; Note that most of the green and red punctae are separate). Although the signal for CNP in neurons is fairly low compared to that of PSD95, its signal strength is always above background level and that the control staining was never punctate (No $1^{\circ} \mathrm{Ab}$ ). Taken to- 

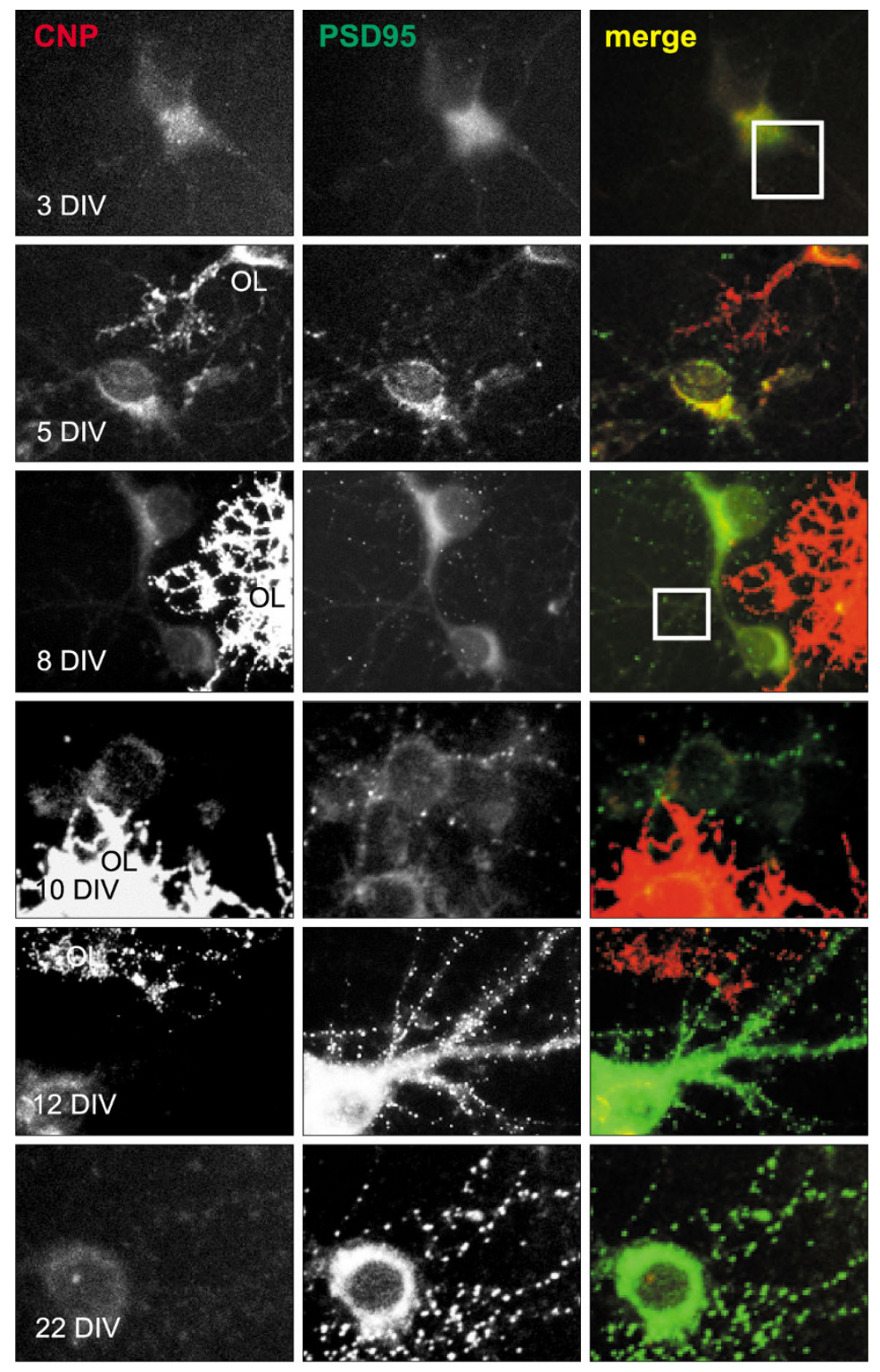

Figure 4. Immunocytochemistry showing transient expression of CNP. Dissociated hippocampal cells were fixed on the indicated DIV and double-stained with affinity-purified rabbit polyclonal $\alpha$-CNP $(1: 500)$ and mouse monoclonal $\alpha$-pan-actin $(1: 200)$. Primary antibodies were visualized by incubating with Alexa488-conjugated goat anti-mouse (green) or Alexa568-conjugated goat anti-rabbit IgG (red). The images shown in Figure 5 are marked by boxed areas in 'merge' panels of 3 and 8 DIV images. Note that weak but punctate CNP stainings appear in 3-12 DIV images. OL, oligodendrocyte. gether, these results indicate that CNP is transiently expressed at low level in developing hippocampal neurons, and that its expression is associated with distinct structure which is different from PSD95containing PSDs.

\section{Expression of CNP in neuronal cell lines}

The finding of CNP expression in neurons is unprecedented. Thus, CNP expression confirmed in neuronal cell lines. Two cell lines were chosen for this purpose; HiB5, a hippocampal stem cell line which has been established from the rat E16 hippocampus (Renfranz et al., 1991) and F11, a hybrid cell line between neuroblastoma N18TG2 and dorsal root ganglionic neurons (Platika et al., 1985). Differentiation of HiB5 and F11 stem cells were induced by platelet- derived growth factor (PDGF) and CAMP, respectively (Cho et al., 2001; Sung et al., 2001), and their homogenates were subjected to immunoblot analysis with polyclonal or monoclonal $\alpha$-CNP antibody. Both antibodies recognized three proteins with apparent molecular weight about 45,46 , and $48 \mathrm{kDa}$ (Figure 6). Comparison with the corresponding bands in the PSD fraction indicated that the uppermost and middle bands corresponded to CNP2 and CNP1, respectively (Figure 6, bars). However, the lowermost band was not present in the PSD fraction from adult rat forebrain. Moreover, it was the major CNP isoform in the F11 cell line (Figure 6, arrowheads). Another 

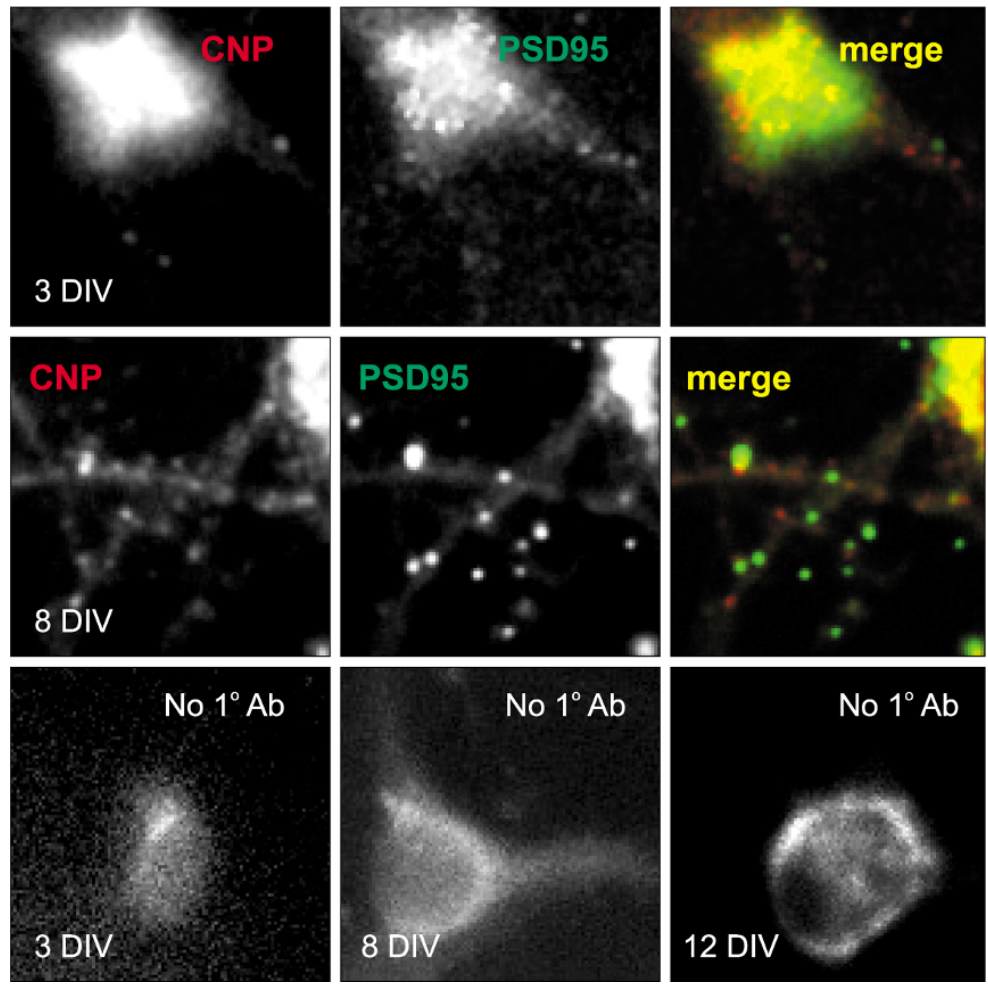

Figure 5. Highly enhanced immunocytochemistry images showing CNP punctae that are distinct from PSD95 ones. The boxed areas in 'merge' panels of 3 and 8 DIV images in Figure 4 were significantly enhanced using the PhotoShop software. Note that most CNP punctae (red) are not superimposed with PSD95 ones (green) in the 'merge' panels. Control images, where primary antibodies are omitted in immunostaining, are shown after similar enhancement of the background signals as the sample stainings. Note that no punctae are evident in these control stainings.
A

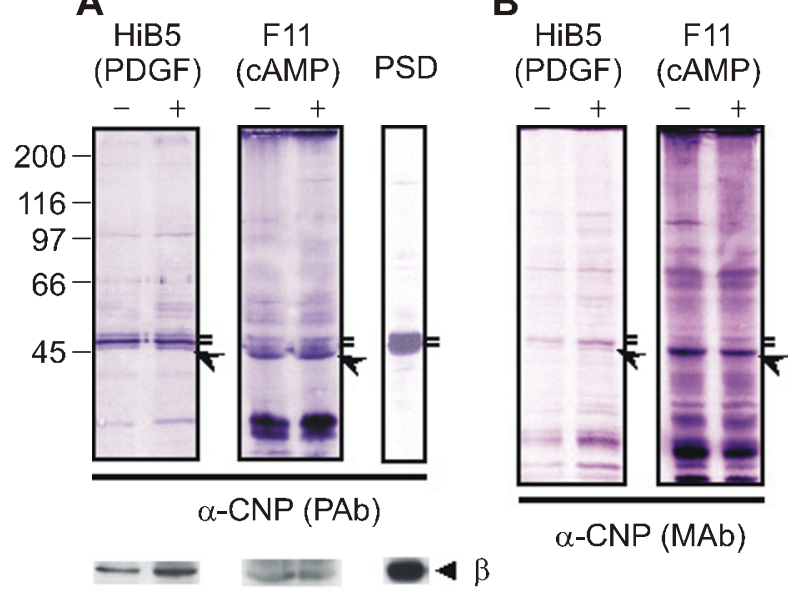

Figure 6. Immunoblot analysis showing expression of CNP in the neuronal stem cell lines. Neuronal stem cell lines, HiB5 and F11, were induced into neuronal morphology by treating with PDGF and CAMP, respectively. Lysates (40 $\mu \mathrm{g}$ each) before and after induction were electrophoresed on an $8 \%$ SDS-gel and subjected to immunoblot analysis with polyclonal (panel A) or monoclonal (panel B) $\alpha-C N P$ antibody. The CNP1 and CNP2 are marked by bars. A newly identified isoform was marked by arrowheads. Note that this new isoform represents the major CNP in F11 cell lines. The expression of $\beta$-tubulin, a neuron-specific protein, is shown at bottom. Molecular sizes are shown in kilodaltons $(\mathrm{kDa})$ on the left. important feature to note is that this pattern of CNP expression is not affected by induction into neuronal morphology, suggesting that CNP is expressed from very early developmental stage.

\section{Discussion}

The CNP is a protein of unknown function that is abundantly expressed in myelinating cells (Scherer et al., 1994; O'Neill et al., 1997). The CNP is also expressed at low level in both neuronal and nonneuronal tissues as described in Introduction. In this report, evidence by immunocytochemistry was presented to support the definitive expression of CNP in hippocampal neurons in culture. The protein, however, was transiently expressed from 3 days to about 2 weeks after plating, a period during which intensive arborization and synaptogenesis occur, implying a function in morphogenesis. The expression of CNP was also confirmed by immunoblots in CNS and PNS stem cell lines, HiB5 and F11. HiB5 is a hippocampal progenitor cell line derived from hippocampal anlage of E16 rat and conditionally immortalized with temperaturesensitive mutant allele of SV40 T antigen, tsA58 (Renfranz et al., 1991). HiB5 cells can differentiate into neuronal cells with neurite-like processes in vitro in serum-free defined $\mathrm{N} 2$ medium containing PDGF at non-permissive temperature of $39^{\circ} \mathrm{C}$ (Cho et al., 
2001; Sung et al., 2001). F11 is a hybrid cell line between neuroblastoma N18TG2 and dorsal root ganglionic neurons (Platika et al., 1985). F11 cells differentiate into neurons in the presence of cAMP or prostaglandins and express neuronal markers including receptor for bradykinin, prostaglandin, opioids and $\mathrm{N}$ - and L-type voltage-dependent $\mathrm{Ca}^{2+}$ channels (Francel et al., 1987; Boland and Dingledine 1990; Fan et al., 1992; Cruciani et al., 1993), suggesting that F11 cells are similar to dorsal root ganglionic neurons. We have shown that both cell lines express CNP even before induction into neuronal morphology, indicating that CNP is expressed from very early developmental stage. Previously, we have shown that CNP is expressed in developing and aberrantly developed Purkinje cells but not in granule cells in cerebellar cell cultures (Cho et al., 2003). Therefore, it is likely that CNP is expressed differentially depending on developmental stages.

CNP isoforms seem to be differentially expressed depending on brain regions and cell types. CNP1 is the major isoform that is expressed in the forebrain (this work). However, comparable amounts of the isoforms were expressed in the cerebellum (Cho et al., 2003), indicating differential expression of each isoform in different brain regions. Furthermore, our results show that there are additional CNP isoforms. Our immunoblot data indicated that there are three isoforms in the HiB5 and F11 stem cell lines. Comparison with the isoforms in the PSD fraction suggests that a newly identified CNP isoform with apparent MW $45 \mathrm{kDa}$ is expressed in both cell lines and is the major isoform that is expressed in the F11 cell lines.

Although CNP is included in the PSD fraction from adult rat forebrain, the CNP is not likely to be specifically associated with the PSD in vivo. The most compelling evidence for this is that the CNP-positive punctae are different from those containing PSD95, a marker for the PSD. Also, both nonionic detergents (OG, Triton) and salt did not efficiently dissociate CNP from the PSD pellet, strongly suggesting that CNP is nonspecifically associated with the PSD during preparation. Then, why is CNP included in the PSD fraction that is a subfraction of the synapse? There are two possibilities. One possibility is that CNP-containing discrete structures may have a similar density to that of PSD, thereby, being co-isolated in the PSD fraction. The other possibility is nonspecific adhesion to the PSD due to very 'sticky' nature of the PSD. Another point to note is that the major tyrosinecontaining protein in CNP-containing band of the onedimensional SDS-gel is not CNP, because immunoprecipitated CNP was only basally phosphorylated on tyrosine.

The function of CNP is still very elusive. It is sug- gested that CNP has a role in morphological changes of cells through modulation of the cytoskeleton, because the activity of CNP is relatively high in tissues containing cells with membranes capable of undergoing transformation and elaboration such as spleen and thymus (Weissbarth et al., 1981), and because CNP induces profound morphological changes in nonneural cells when expressed ectopically (Staugaitis et al., 1990; De Angelis and Braun, 1994). Our finding that CNP is expressed in the early stage of morphological differentiation compromises well with this suggested role. Recently, Cnp1, a gene that encodes both CNP1 and CNP2 in oligodendrocytes, was found to be essential for axonal survival but not for myelin assembly by Lappe-Siefke et al. (2003). They found that Cnp1 knockout mice developed axonal swellings and neurodegeneration throughout the brain, but the ultrastructure, periodicity and physical stability of myelin were not altered in these mice. These pathologies could occur solely by defects in OL. However, our findings suggest that it could well be due to a defect of communication between myelinating glia and neurons.

\section{Acknowledgment}

This work was supported by grant no. R02-200000140 from the Basic Research Program of the Korea Science \& Engineering Foundation.

\section{References}

Bifulco M, Laezza C, Stingo S, Wolff J. 2',3'-Cyclic nucleotide 3'-phosphodiesterase: a membrane-bound, microtubule-associated protein and membrane anchor for tubulin. Proc Natl Acad Sci USA 2002;99:1807-12

Boland LM, Dingledine R. Multiple components of both transient and sustained barium currents in a rat dorsal root ganglion cell line. J Physiol 1990;420:223-45

Brewer GJ, Torricelli JR, Evege EK, Price PJ. Optimized survival of hippocampal neurons in B27-supplemented Neurobasal, a new serum-free medium combination. J Neurosci Res 1993;35:567-76

Carlin RK, Grab DJ, Cohen RS, Siekevitz P. Isolation and characterization of postsynaptic densities from various brain regions: enrichment of different types of postsynaptic densities. J Cell Biol 1980;86:831-43

Cho JH, Kwon IS, Kim S, Ghil SH, Tsai MJ, Kim YS, Lee $Y D$, Suh-Kim H. Overexpression of BETA2/NeuroD induces neurite outgrowth in F11 neuroblastoma cells. J Neurochem 2001;77:103-9

Cho K-O, Hunt CA, Kennedy MB. The rat brain postsynaptic density fraction contains a homolog of the Drosophila discs-large tumor suppressor protein. Neuron 1992;9:929-42

Cho S-J, Jung JS, Jin I, Moon IS. 2',3'-Cyclic Nucleotide 
3'-Phosphodiesterase is Expressed in Dissociated Rat Cerebellar Cells and Included in the Postsynaptic Density Fraction. Mol Cells 2003;16:128-35

Cruciani RA, Dvorkin B, Morris SA, Crain SM, Makman MH. Direct coupling of opioid receptors to both stimulatory and inhibitory guanine nucleotide-binding proteins in F-11 neuroblastoma-sensory neuron hybrid cells. Proc Natl Acad Sci USA 1993;90:3019-23

De Angelis DA, Braun PE. Isoprenylation of brain 2',3'-cyclic nucleotide 3'-phosphodiesterase modulates cell morphology. J Neurosci Res 1994;39:386-97

Dreiling CE, Schilling RJ, Reitz RC. 2',3'-Cyclic nucleotide 3'-phosphohydrolase in rat liver mitochondrial membranes. Biochim Biophys Acta 1981;640:114-20

Fan SF, Shen KF, Scheideler MA, Crain SM. F11 neuroblastoma DRG neuron hybrid cells express inhibitory muand delta-opioid receptors which increase voltage-dependent K+ currents upon activation. Brain Res 1992;590:329-33

Francel PC, Miller RJ, Dawson G. Modulation of bradykinininduced inositol triphosphate release in a novel neuroblastoma dorsal root ganglion sensory neuron cell line (F11). J Neurochem 1987;48:1632-9

Giulian D, Moore S. Identification of 2',3'-cyclic nucleotide 3'-phosphodiesterase in the vertebrate retina. J Biol Chem 1980;255:5993-5

Kennedy MB. The postsynaptic density at glutamatergic synapses. Trends Neurosci 1997;20:264-8

Kennedy MB. Signal transduction molecules at the glutamatergic postsynaptic membrane. Brain Res Brain Res Rev 1998;26:243-57

Kennedy MB. Signal-processing machines at the postsynaptic density. Science 2000;290:750-4

Laezza C, Wolff J, Bifulco M. Identification of a $48-\mathrm{kDa}$ prenylated protein that associates with microtubules as 2',3'-cyclic nucleotide 3'-phosphodiesterase in FRTL-5 cells. FEBS Lett 1997;413:260-4

Lappe-Siefke C, Goebbels S, Gravel M, Nicksch E, Lee J, Braun PE, Griffiths IR, Nave KA. Disruption of Cnp1 uncouples oligodendroglial functions in axonal support and myelination. Nat Genet 2003;33:366-74

McFerran B, Burgoyne R. 2',3'-Cyclic nucleotide 3'-phospho- diesterase is associated with mitochondria in diverse adrenal cell types. J Cell Sci 1997;110:2979-85

O'Neill RC, Minuk J, Cox ME, Braun PE, Gravel M. CNP2 mRNA directs synthesis of both CNP1 and CNP2 polypeptides. J Neurosci Res 1997:50:248-57

Platika D, Boulos MH, Baizer L, Fishman MC. Neuronal traits of clonal cell lines derived by fusion of dorsal root ganglia neurons with neuroblastoma cells. Proc Natl Acad Sci USA 1985;82:3499-503

Renfranz P J, Cunningham MG, McKay RD. Region-specific differentiation of the hippocampal stem cell line HiB5 upon implantation into the developing mammalian brain. Cell 1999;66:713-29

Scherer SS, Braun PE, Grinspan J, Collarini E, Wang DY, Kamholz J. Differential regulation of the 2',3'-cyclic nucleotide 3'-phosphodiesterase gene during oligodendrocyte development. Neuron 1994;12:1363-75

Siekevitz P. The postsynaptic density: a possible role in long-lasting effects in the central nervous system. Proc Natl Acad Sci USA 1985;82:3494-8

Staugaitis SM, Bernier L, Smith PR, Colman DR. Expression of the oligodendrocyte marker 2',3'-cyclic nucleotide 3'- phosphodiesterase in non-glial cells. J Neurosci Res 1990;25: $556-60$

Sung JY, Lee SY, Min DS, Eom TY, Ahn YS, Choi MU, Kwon YK, Chung KC. Differential activation of phospholipases by mitogenic EGF and neurogenic PDGF in immortalized hippocampal stem cell lines. J Neurochem 2001; 78:1044-53

Tsukada $Y$, Kurihara T. In Myelin: Biology and Chemistry (Martenson RE, ed), 1992, 449-480, CRC Press, Inc., Boca Raton, FL Uyemura K, Tobari C, Hirano S, Tsukada Y. Comparative studies on the myelin proteins of bovine peripheral nerve and spinal cord. J Neurochem 1972;19:2607-14

Vogel US, Thompson RJ. Molecular structure, localization, and possible functions of the myelin-associated enzyme 2',3'-cyclic nucleotide 3'-phosphodiesterase. J Neurochem 1988:50:1667-77

Weissbarth S, Maker HS, Raes I, Brannan TS, Lapin EP, Lehrer GM. The activity of 2',3'-cyclic nucleotide 3'-phosphodiesterase in rat tissues. J Neurochem 1981;37:677-80 